\title{
DEVELOPING A MOBILE SOCIAL LEARNING APPLICATION FOR INTERDISCIPLINARY TECHNOLOGY COURSES
}

\author{
Brian Thoms ${ }^{1}$ and Evren Eryilmaz ${ }^{2}$ \\ ${ }^{1}$ CSU Channel Islands, Camarillo, California, USA \\ ${ }^{2}$ CSU Sacramento, Sacramento, California, USA
}

\begin{abstract}
Smartphone adoption has grown steadily and now represents roughly 50\% of all Internet traffic. Advances in mobile application development along with its widespread adoption offers unique opportunities within computing education to quickly offer knowledge and information directly into the hands of the learner. Adopting a mobile-first approach, this paper introduces the construction of a mobile social learning platform, built on top of existing social learning software and measures its adoption in interdisciplinary computing courses. The goal of the mobile app is to complement the browser-based system and provide access to course notifications, gradebook and discussion posts. Initial results found 50\% of students adopting the smartphone app regularly, while most students preferred to use the browser-based system. Students adopting the mobile application indicated that they liked the ability to view course notifications, assignments, grades and instructor feedback from within the mobile app.
\end{abstract}

\section{KEYWORDS}

Computing Education, Social Networking, Mobile Application

\section{INTRODUCTION}

Across North America, colleges and universities are facing significant increases in enrollment in undergraduate computing courses and programs (Camp et al., 2017). This trend reflects projections produced by the U.S. Bureau of Labor Statistics (Bureau of Labor Statistics, Occupational Projections, 2020), which cites substantial increases in opportunities over the next ten years in various areas of computing, such as software developers and information security analysts $(25 \%$ and $31 \%$ respectively). Interest in CS by non-majors has also substantially increased (Camp et al., 2017) as students attempt to build technical skills that are transferrable into the workplace (Camp et al., 2015). In some cases, this has led to CS majors taking courses along-side non-CS majors. This interdisciplinary approach to CS education works particularly well when natural synergies and overlap exist in the CS and non-CS curriculum.

During this same timeframe, the world has seen a steady rise in smartphone adoption and mobile computing, specifically with regards to accessing online content. Today, smartphones comprise over $50 \%$ of all Internet traffic (Clement, 2019). Consequently, in order to support the fact that students are using mobile technologies, they require tools on such devices to support individual learning, as identified in (Gikas and Grant, 2013). Yet delivery of effective education content over mobile devices remains challenging and face added workloads and costs on increasingly taxed educational systems.

In this paper, we introduce mobile social learning software, built atop an existing web-based social learning platform. More specifically, this paper describes the construction of a cross-platform mobile application for iOS and Android, and its implementation across interdisciplinary technology courses. The goal of this system is to offer students with a mobile-first system for instructional content and course features such as notifications, assignments, grades and peer-interactions. Initial results found that the mobile app, while useful as an informational resource was less utilized than the browser-based learning environment. This paper chronicles this experience. 


\section{BACKGROUND}

\subsection{Course Design}

The Computer Science course COMP449 - Human Computer Interaction (HCI), studies the ways in which people interact with computing devices and design technologies to support interaction with these devices in novel ways. The course is cross-listed with Psychology and serves as an undergraduate upper-division general education course required for graduation. Cross-listed computing courses are becoming more popular and offer unique opportunities for computing programs and introduce more reluctant students to areas of computing through the integration of theories and concepts of other disciplines (Carter, 2014). In 2020, the software for COMP449 was enhanced with a mobile application, to provide students with a mobile-first alternative to accessing the gradebook, quizzes, discussion-board and blogs. In Version 1, the goal was to offer a complementary app to the web-based system and to measure students' satisfaction with the system.

\subsection{SocialXYZ OSN Design}

Constructivist learning places individuals at the center of the learning experience (Piaget, 1952). Instructional software embracing this approach often leverages social components common in online social networking (OSN) software (Thoms, Garrett and Ryan, 2009), (Boulos, Maramba and Wheeler, 2006) that have been shown to elevate learning, software satisfaction and course satisfaction, compared to traditional learning management systems (Thoms and Eryilmaz, 2014).

The primary system in this paper is SocialXYZ, which is software built atop Elgg, an open source social networking engine. Designed specifically for browser-based interaction, Elgg comes bundled with online discussion boards, blogs, file gallery, profile building and peer-to-peer connection-making. Illustrated in Figure 1 , the course landing page highlights content from across the site including assignments and provides quick access to the course gradebook, syllabus, files and peer-connections. Illustrated in Figure 2 is the center of student-to-student interaction, the asynchronous online discussion board. Critical to SocialXYZ, the discussion board facilitates cognitive, on-topic, on-task, and sustained discussion among students (Pituch and Lee, 2006), and allows peers to communicate using similar language styles (Caldwell, 2007). In previous design iterations, SocialXYZ was enhanced to include a recommender system based on the sentiment and quality of discussion posts (Thoms et al., 2020), (Thoms et al., 2017). While effective in the browser, the system interface breaks down across mobile devices, where screen real estate is constrained. In this paper, we attempt to improve the user experience across mobile devices.

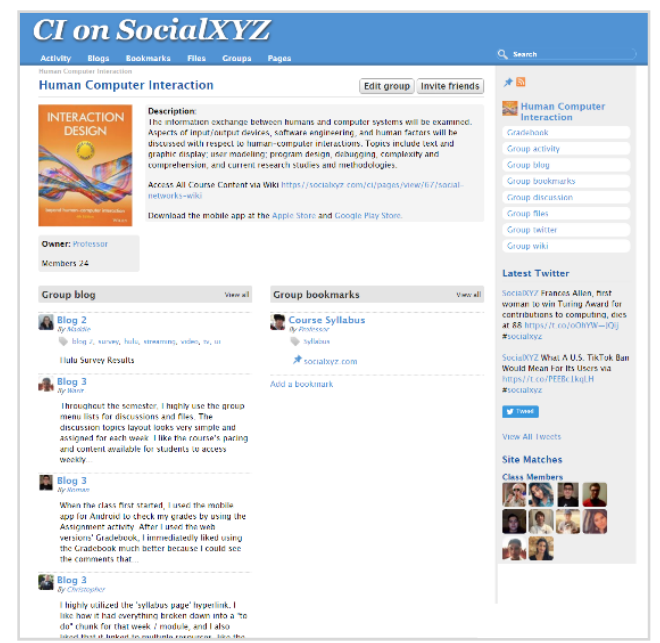

Figure 1. SocialXYZ Landing Page



Figure 2. SocialXYZ AOD 


\subsection{SocialXYZ Mobile App Design}

Built for Android and iOS, a mobile app was developed using the React Native JavaScript programming language and leverages a REST architecture for backend processing. Version 1 of the system includes critical features including course announcements, syllabus, discussion board and gradebook. The Home screen, which is illustrated in Figure 3, provides access to this primary application feature-set. Course assignments, depicted in Figure 4, also provides functionality for grades and instructor feedback. The threaded asynchronous online discussion board is illustrated in Figure 5 and Figure 6 illustrates the screen for a typical discussion response. Response metrics highlighted in Figure 6 were studied in previous design iterations of the web-based platform (see [Thoms et al., 2020], [Thoms et al., 2017]). Enhanced mobile app features leveraged native smartphone functionality such as automated delivery of system notifications and gesture-based screen swiping.

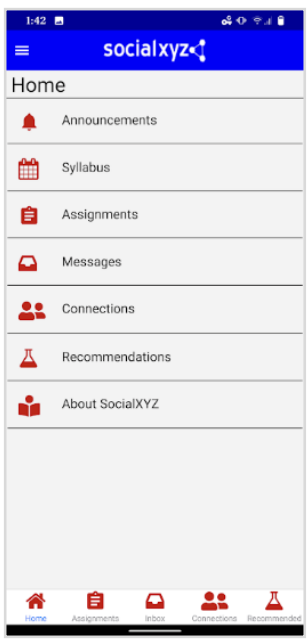

Figure 3. Home Screen

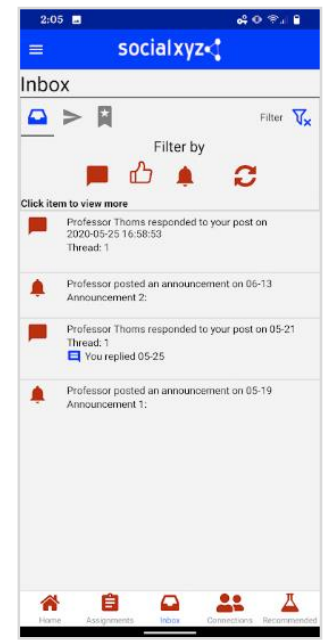

Figure 7. Inbox

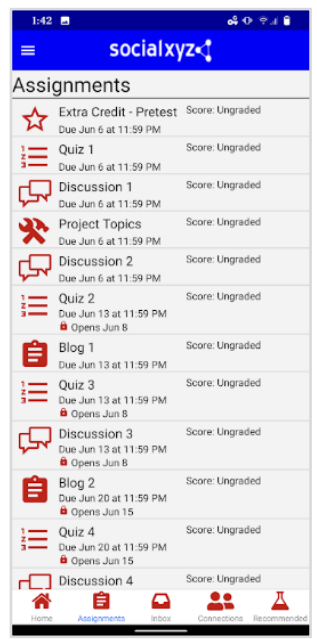

Figure 4. Assignments

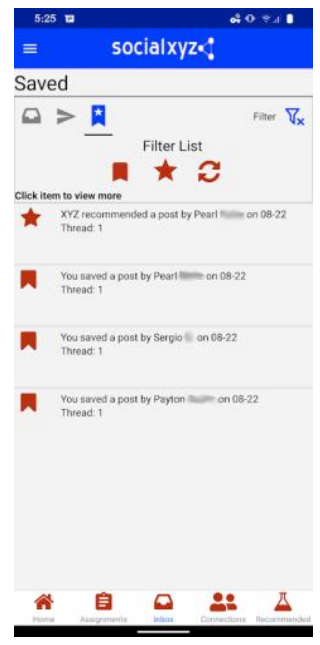

Figure 8. Bookmarks

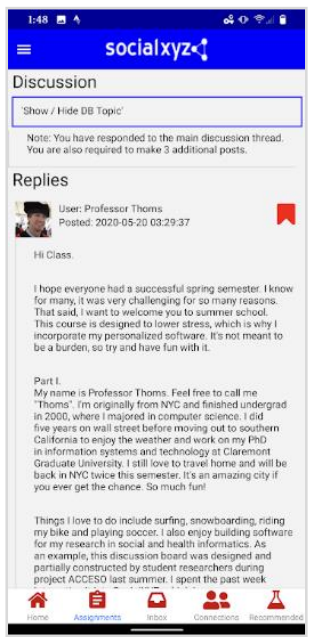

Figure 5. Discussion Board

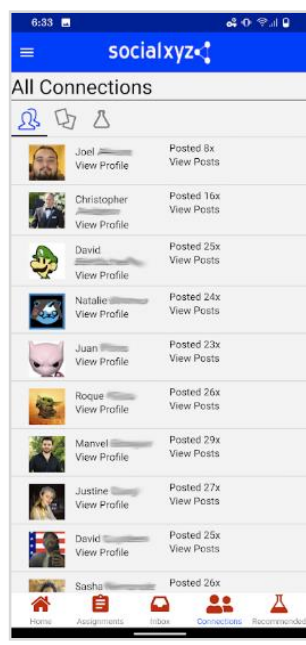

Figure 9. Connections

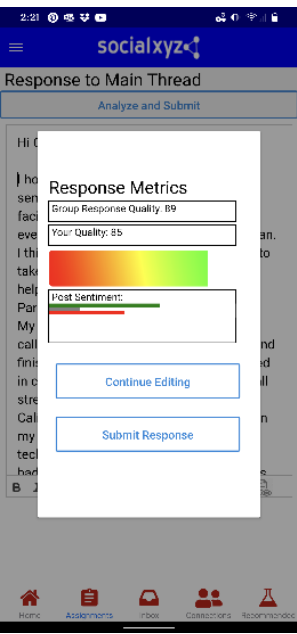

Figure 6. Response Screen

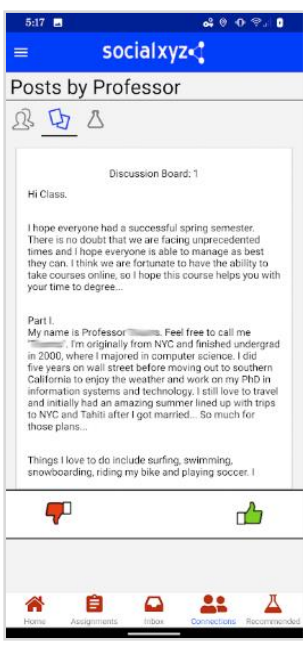

Figure 10. User Posts

Figure 7 and Figure 8 illustrate app messages, announcements and bookmarks. All content created by or directed to a user can be found in the user's Inbox, Sent Box and Bookmarks screens. Figure 9 and Figure 10 illustrate screens for direct peer-to-peer interactions across the system. In short, users may quickly navigate to peer content via the Connections screen, where they can thumbs-up / thumbs-down content, which is saved directly to their Inbox. System Recommendations are available through the 'flask' icon and highlight top-rated content from across the system. 


\section{RESULTS}

In summer of 2020, the system was tested across an online, asynchronous upper-division technology course, serving students looking to satisfy general education requirements for graduation. A total of 20 students participated in the course.

\subsection{System Usage}

Broken down in Figure 11, the composition of the course was $60 \%$ computer science and information technology majors and $40 \%$ non-computing majors including psychology, business and health sciences. Anonymous pretest data identified $100 \%$ of students having experience with learning management software such as Blackboard or Canvas and $91 \%$ having experience with social networking technologies such as Facebook and Twitter.

The total number of web views accessed through the browser-based system was 8,874 , while the number of API calls from all mobile devices was 2,655. For results on web-based usage, we refer you to previous research in (Thoms et al., 2007). An analysis of device operating systems found that $65 \%$ of users used the app on iOS devices and 35\% used the app on Android devices. All students attempted to use both the website and the mobile app initially, while the browser-based system was used considerably more and accounted for $75 \%$ of total interactions across the system. Posttest survey data identified $50 \%$ of respondents indicating that they used the mobile application frequently. Detailed in Figure 12, an analysis of API log files found that the primary use of the mobile app was largely informational. Viewing mobile messages and announcements accounted for $40 \%$ of all API calls. An additional $29 \%$ of API calls accessed the discussion board and system recommendations. Assignments and grades accounted for $13 \%$ of API calls as did API calls for peer-to-peer connections, which was also $13 \%$. User generated content represented a much smaller percentage of overall interactions with the system. $3 \%$ of API calls made added discussion board posts and $3 \%$ of API calls made accessed the mobile quizzes. In total, the mobile discussion board was accessed 358 times, while the browser-based discussion board was accessed 791 times.

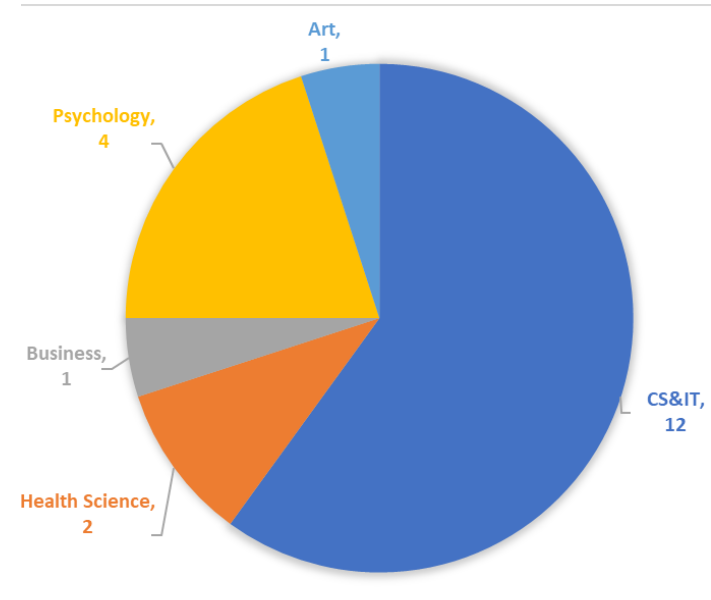

Figure 11. System Demographics

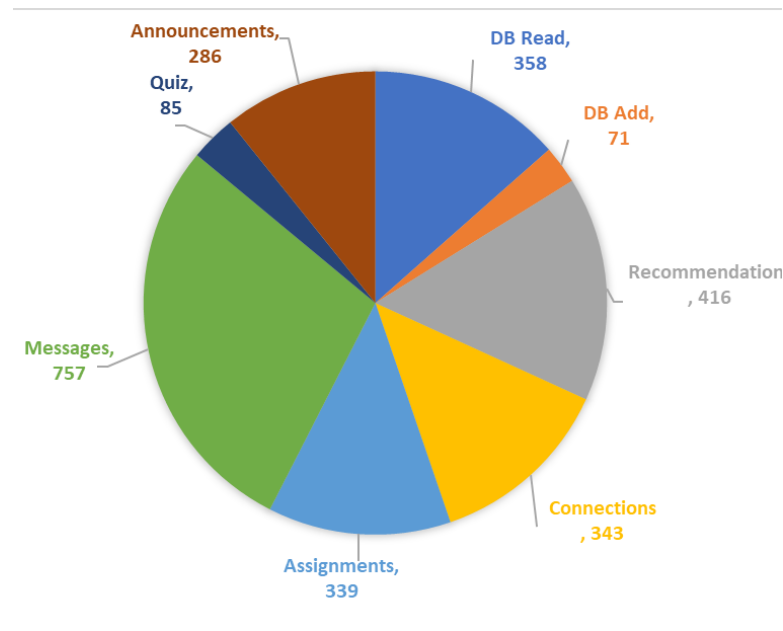

Figure 12. Mobile App Usage by REST Call

\subsection{System Satisfaction}

Detailed in Table 1, posttest survey data helped to gauge general satisfaction with the mobile app. Of the 18 students responding to the posttest, $78 \%$ indicated satisfaction with the mobile application compared to $100 \%$ indicating satisfaction with the browser-based system. More specifically related to the mobile app, 50\% stated that it was easy to use and 50\% stated it as an excellent way to receive updates from the instructor. Additionally, $50 \%$ of respondents indicated that the mobile app was an excellent way to view updates from their classmates. It was found that $28 \%$ of respondents strongly disagreed that the mobile app was an excellent way to post to the discussion with only $22 \%$ of respondents agreeing with this statement. 
Table 1. Posttest Survey Results

\begin{tabular}{|c|c|c|c|c|c|}
\hline Posttest Questions $(n=18)$ & SA & $\mathbf{A}$ & $\mathbf{N}$ & D & SD \\
\hline I used Mobile SocialXYZ frequently. & 2 & 7 & 5 & 2 & 2 \\
\hline I preferred to use Mobile SocialXYZ over the Website. & 3 & 1 & 6 & 6 & 2 \\
\hline Mobile SocialXYZ was easy to use. & 2 & 7 & 6 & 3 & 0 \\
\hline Mobile SocialXYZ provided useful functionality. & 3 & 5 & 8 & 2 & 0 \\
\hline Mobile SocialXYZ was an excellent way to view course content. & 5 & 2 & 7 & 4 & 0 \\
\hline Mobile SocialXYZ was an excellent way to view updates by the instr & 5 & 4 & 9 & 0 & 0 \\
\hline Mobile SocialXYZ was an excellent way to view updates by my classmates. & 5 & 4 & 8 & 1 & 0 \\
\hline Mobile SocialXYZ was an excellent way to post to the discussion. & 2 & 2 & 9 & 3 & 2 \\
\hline
\end{tabular}

\section{CONCLUSION}

This experiential paper introduces the design, development and implementation of Mobile SocialXYZ built atop existing open source social networking software. Overall, the mobile app complemented the browser-based system providing students with a mobile-first approach to accessing content such as announcements, gradebook and discussion posts and provides students with features for interacting with their peers through social networking and notifications. Additionally, this paper presents on Version 1 of the mobile app. New features for direct messaging, AWS cloud storage integration and an advanced content-based recommendation system are under construction.

\section{REFERENCES}

Boulos, M., Maramba, I., Wheeler, S. (2006). "Wikis, blogs and podcasts: a new generation of web-based tools for virtual collaborative clinical practice and education," BMC Medical Education, 6(41).

Bureau of Labor Statistics, Occupational Projections (2020). Retrieved online on June 21, 2020 from https://data.bls.gov/projections/occupationProj.

Caldwell, JE. (2007). "Clickers in the Large Classroom: Current Research and Best-practice Tips", CBE-Life Sciences Education, 6(1).

Camp, T., Adrion, W.R., Bizot, B., Davidson, S., Hall, M., Hambrusch, S., Walker, E. and Zweben, S. (2017). Generation CS: The Growth of Computer Science. ACM Inroads 8(2), pp. 44-50.

Camp, T., Zweben, S., Walker, E., and Barker, L. (2015). Booming enrollments: Good times? In Proceedings of the 46th ACM Technical Symposium on Computer Science Education (Kansas City, United States, (March 04-07, 2015). SIGSCE '15. ACM, New York, NY, 80-81.

Carter, L. (2014). "Interdisciplinary computing classes: worth the effort," In Proceedings of the 45th ACM technical symposium on Computer science education (SIGCSE '14). ACM, New York, NY, USA.

Clement, J. (2019). "Percentage of all global web pages served to mobile phones from 2009 to 2018," Statistica. Retrieved online from https://www.statista.com/statistics/277125/share-of-website-traffic-coming-from-mobile-devices/ on August 20, 2020.

Gikas, J., Grant, MM. (2013). "Mobile computing devices in higher education: Student perspectives on learning with cellphones, smartphones \& social media," The Internet and Higher Education, v19, pp 18-26.

J. Piaget, The Origins of Intelligence in Children. New York, NY: International University Press, 1952.

Pituch, K., Lee, Y. (2006). "The influence of system characteristics on e-learning use," Computers \& Education, 47(2).

Thoms, B., Eryilmaz, E. (2014). "How Media Choice Affects Learner Interactions in Distance Learning Classes," Computers \& Education, v75.

Thoms, B., Eryilmaz, E. Dubin, N. Hernandez, R. Colon-Cerezo, S. (2020). "Real-Time Visualization to Improve Quality in Computer Mediated Communication,” Web Intelligence Journal, v18(1), 2020.

Thoms, B., Eryilmaz, E. Mercado, G. Ramirez, B. Rodriguez, J. (2017). "Towards A Sentiment Analyzing Discussion Board," Hawaiian International Conference on System Sciences (HICSS 50).

Thoms, B., Garrett N. and Ryan, T. (2009). "Online Learning Communities in the New 'U'. International Journal of Networking and Virtual Organisations, 6(5), pp. 499-517.

Thoms, B., Garrett, N., Soffer, M. and Ryan, T. (2007). "Resurrecting Graduate Conversation through an Online Learning Community", IADIS Multi Conference on Computer Science and Information Systems (IADIS-2007). 